\section{A GEOSTRESS MEASURE- MENT METHOD BASED ON AN INTEGRATED DRILLING AND OPTICAL MICROSCOPIC IMAGING SYSTEM}

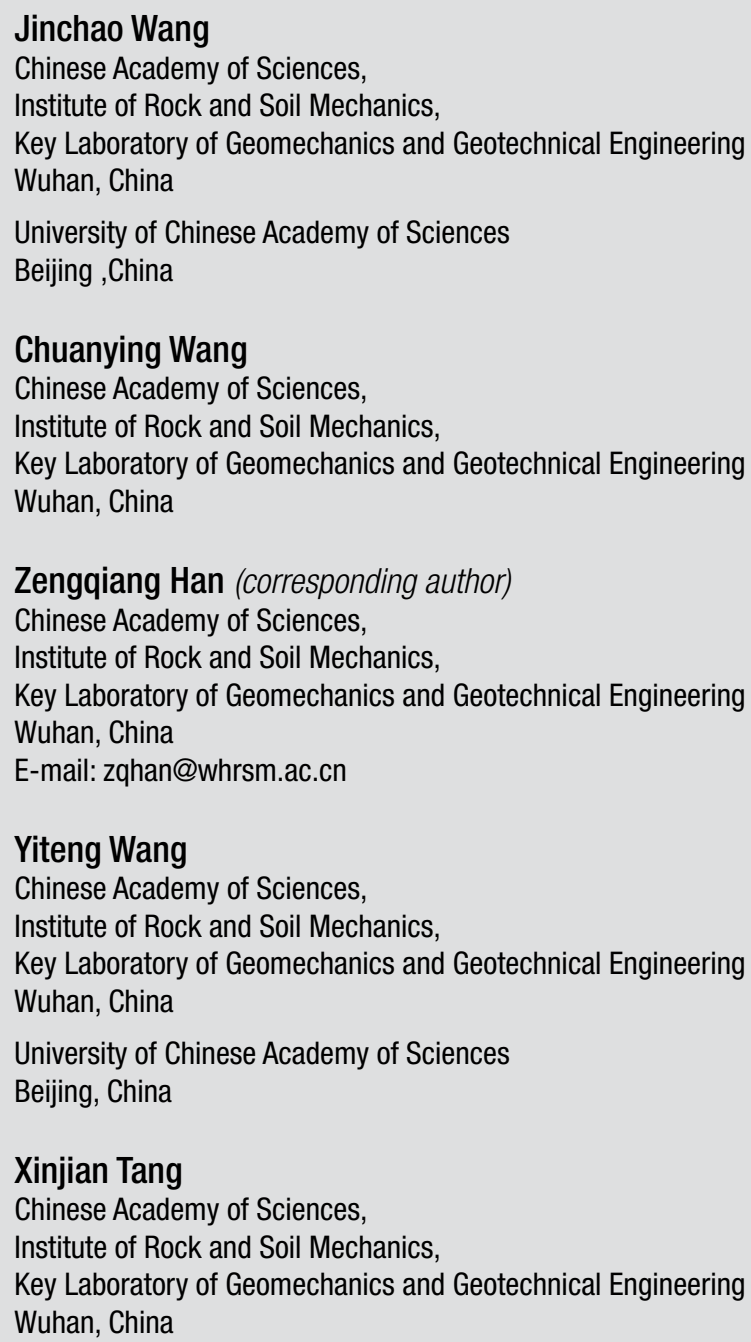

\section{Xinjian Tang}

Chinese Academy of Sciences, Institute of Rock and Soil Mechanics, Key Laboratory of Geomechanics and Geotechnical Engineering Wuhan, China

\section{Keywords}

geostress; probe; optical microscopy; measurement while drilling; search circle

DOI https://doi.org/10.18690/actageotechslov.15.1.17-27.2018

\section{Abstract}

Conventional geostress measurement methods are limited by deficiencies including the measurable depth, the complexity, and the long duration of operation. To address these problems and achieve the measurement of geostress in deep wells under conditions of complex high pressures and high temperatures, we propose a new measurement method for geostress based on an integrated drilling and optical microscopy system. Its innovative integrated structure eliminates the problems associated with complex procedures and depth limits, and avoids rock creep caused by long delays, significantly improving the accuracy and range of the measurements. It works by using microscopic imaging and direct contact probes to capture the changes of a borehole's cross-sectional outlines before and after stress relief. The resulting images are analyzed with search circles to obtain the positions of probe apices, which can be fitted into ellipses that describe the outlines, and calculate the state of the stress. The validity and accuracy of the method was verified by in-door tests and field applications in the ZK1 borehole. The results show that: (1) the integrated system can be used to measure micrometer-grade deformations; (2) the searchcircle approach can accurately obtain the positions of probe apices; and (3) the stress measurement method based on the system is accurate and feasible.

\section{INTRODUCTION}

Geostress, while a universal phenomenon, has been difficult to assess and measure. As we continue our exploration into the deeper crusts, their stresses will play an increasingly greater role in the design and decisionmaking of our deep engineering projects. Work on the theory and practice of stress measurements has led to the creation of new techniques and instruments. The most typical such device was the USBM gauge, developed by the U.S. Bureau of Mines in the 1950s, and the stress-relief method it represents has remained the most commonly used approach $[1,2,3]$.

The stress-relief method is based on the theory of elasticity. It assumes a homogeneous and continuous rock mass, and the same stress-strain function when both loading and unloading stress. It also considers the rock elements being removed as having negligible weight (gravity stress) compared to their load. The measurements are usually made with the following steps:

1) A large borehole is drilled to a predetermined depth close to the measurement position;

2) A smaller hole concentric with the borehole is drilled into the bottom, and three-axis strain gauges (for 
measuring wall strains) or deformation transducers (for diameter deformations) are installed into the hole;

3) The larger borehole is then extended down, separating the now pipe-like core from the base rock to relieve the stress.

This conventional method involves a series of complicated tasks: drilling the large well, leveling the bottom, shaping the pilot hole, drilling the measurement hole, installing transducers, and drilling the relief well. In this process, the drilling rod must be lifted several times from the well, have the drill replaced, and lower back to the bottom. The usefulness of the method in deeper wells is further impeded by the lengths of the transducer cables. For these reasons the utilization of the stressrelief method has often been restricted to the testing of shallow wells $[4,5]$.

Another problem stems from the time delays in measurements. The duration from the installation of the transducers to the end of the stress relief lasts a few days at least, and the test process lasts several tens of minutes by itself. The delays are long enough for rock creep to significantly alter the body's mechanical parameters, deviating from the assumed elastic relationships $[6,7]$. The resulting stress data are seldom very accurate for this reason [8].

To address these common issues, this paper introduces an integrated drilling and optical microscopic measurement system. It incorporates an outer drill and an inner drill, respectively, for relief and measurement holes. A control module connects and disconnects the drills as needed, replacing the complicated traditional approach. An optical module is used to directly acquire deformation data from the walls. This system can be utilized in deep wells, mitigates the delay-induced errors by real-time measurements, and effectively solves the major technical issues encountered in stress relief. A stress-measurement method based on the system is also introduced.

\section{INTEGRATED DRILLING AND OPTICAL MICROSCOPIC MEASUREMENT SYSTEM}

The system can be divided into the drilling and acquisition sub-systems, the former for drilling the measurement and relief holes, and the latter for acquiring and recording the borehole's diameter deformations.

\subsection{Theory}

The integrated system is still based on the principles of stress relief. It measures the deformations of the rock body before and after the stress relief, and extrapolates the initial stress from them in the following steps:

1) The integrated system drills the measurement hole, and lowers the optical module to the measurement position (see Fig. 1(a));

2) After the optical module arrives at the position, the control module separates the inner and outer drills, the inner drill stays at rest, while the outer drill resumes drilling (see Fig. 1(b));

3) The outer drill overtakes the measurement hole, separating the pipe-like core from the rock mass (see Fig. 1(c)), which relieves the stress around the optical module;

4) The optical module detects the minuscule diameter changes, which is used to compute the deformation of the cross-section and its horizontal stress;

5) Measurements are made in three intersecting boreholes to determine the three-dimensional state of stress $[9,10,11,12]$.

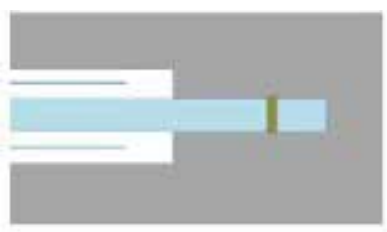

a)

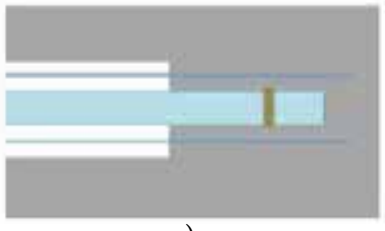

c)

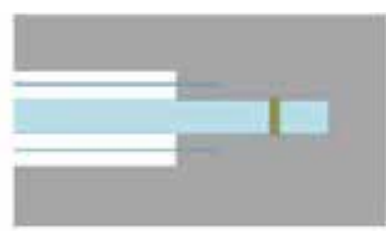

b)

Figure 1.

Measurement process:

(a) drilling the measurement hole;

(b) drilling the relief hole;

(c) stress relief.

\subsection{Structure of the drilling system}

The drilling system consists of the outer drill, the control module, and the inner drill, shown in Fig. 2. The inner drill is used to create the measurement hole, and lower the optical microscopic component to the measurement position. After the preset bottom of the measurement hole is reached, the control module will decouple the two drills, and the inner drill will be stationary relative to the surrounding rock body, allowing the acquisition system inside to perform its measurements, while the outer drill continues drilling downwards to create the relief well. As the relief well takes shape, stress can be completely relieved from the core, which is detected by the optical module. At the end of the entire operation, all the system components can be retrieved together. 


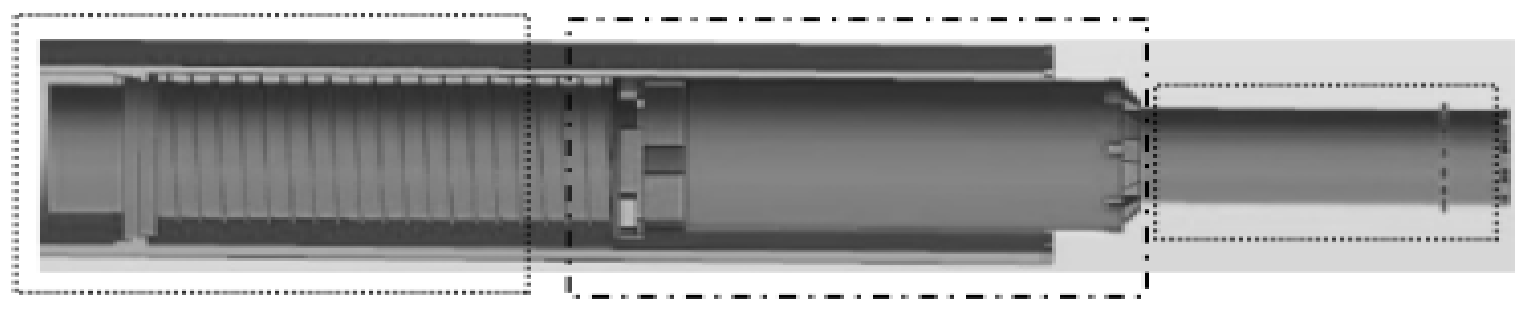

(a) Outer drill (relief)

(b) Control module (decoupling)

(c) Inner drill (measurement)

Figure 2. Structure of the drilling system.

\subsection{Structure of the acquisition system}

The acquisition system consists of a number of probes, a camera, and an electronic compass, shown in Fig. 3. As the surrounding rock core becomes separated from the larger rock mass, it is relieved of the rock mass' stresses, causing the measurement hole to deform. Each probe is in direct contact with the walls of the hole via a prod protruding from the drill, allowing the deformation to be recorded in real time.

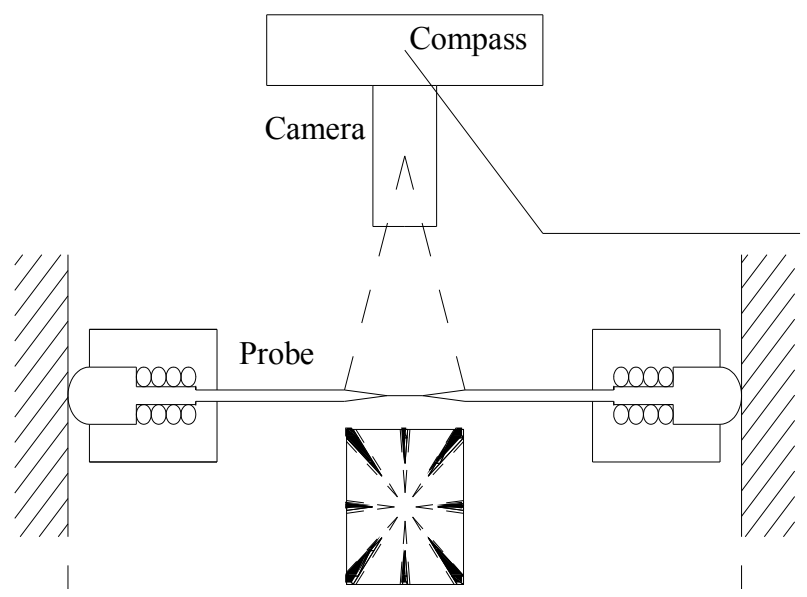

Figure 3. Structure of the acquisition system.

The changing positions of the probes are captured by the camera, consisting of a fixed-focus lens and a microscopic CCD component (see Fig. 4). Since the target area is small, a lens with a small FOV is chosen. Since the CCD image has a greater width than its height, the shorter side is used to determine its physical resolution, which is the physical length of the side divided by its number of pixels, e.g., 576px for a standard capturing component with a resolution of $768^{\star} 576$. The device used in this study captures a 500px-tall image from a $3.5 \mathrm{~mm}$-tall area, meaning the image has a physical resolution of $0.007 \mathrm{~mm}$.
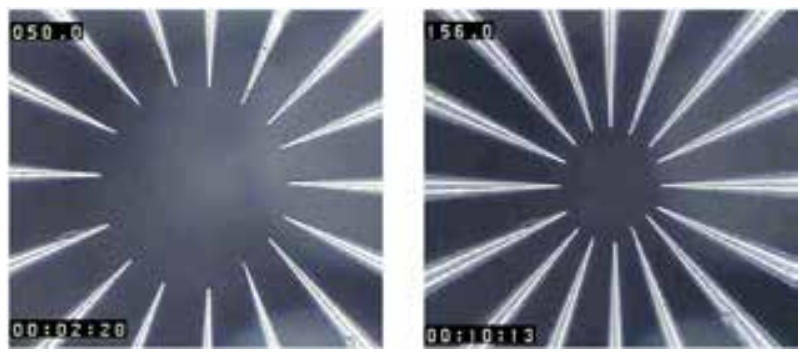

Figure 4. Image data from the probes.

The directional data of the deformation are an important parameters that cannot be accurately recorded by the images. Hence, an electronic compass is used, with its north set to be the same direction as one of the probes. The direction of each probe can then be derived.

\subsection{Advantages of the system}

The proposed system has the following standout features. Firstly, its deployment is vastly simpler than the conventional method. This is accomplished by using a control module to coordinate the movements of the outer and inner drills, integrating the functions of downward drilling, borehole shaping, and measurement in one device. Additionally, the measurement module can start working as soon as the measurement hole is formed, and continue its recording throughout the relief operation, generating more valid data. By eliminating the long delay that comes from replacing drills, the influence of rock creep can be minimized, and the resulting errors can be avoided. This means that the system is more efficient, and less vulnerable to external disturbances than conventional devices.

Secondly, the system uses probes in direct contact with the walls, captures their deformation with microscopic imaging, calculates the borehole's deformation after relief from the images, and determines the stress from the deformation. The microscopic imaging allows accuracy at the micrometer level; the probes are made with 
materials resistant to abrasion, ensuring the system can be operational under unusually harsh conditions. These features give the system high accuracy and adaptability, and present a solution to the limited reach and accuracy in conventional stress-relief methods.

\section{ACQUISITION OF DEFORMATION DATA}

From the image data, it is possible to directly identify the positions of the probes' sharp apices by computer. However, for our required accuracy, this is a process prone to errors. In this study, the images are preprocessed, allowing the probe edges to be detected. The precise coordinates of the apices are then determined as the intersections of these edge lines.

\subsection{Pre-processing}

The inside of a borehole is not a homogeneous environment. The image data can be affected by the noises from external signals and a minor turbidity in the liquid between the camera and the probes, as seen in Fig. 5(a). Since the adaptive median filter (AMF) algorithm can preserve the edges in an image, it is chosen for the de-noise operation. AMF functions by adjusting the filter window based on noise density, using different methods to process noise and signal pixels, maintaining the gray-scale values of the signals, while applying a median filter to the noises.

Let the grayscale of the pixel $(x, y)$ be $f_{x y}$, the current work window be $A_{x y}$, and the preset largest window permitted be $A$, in which the minimum, median and

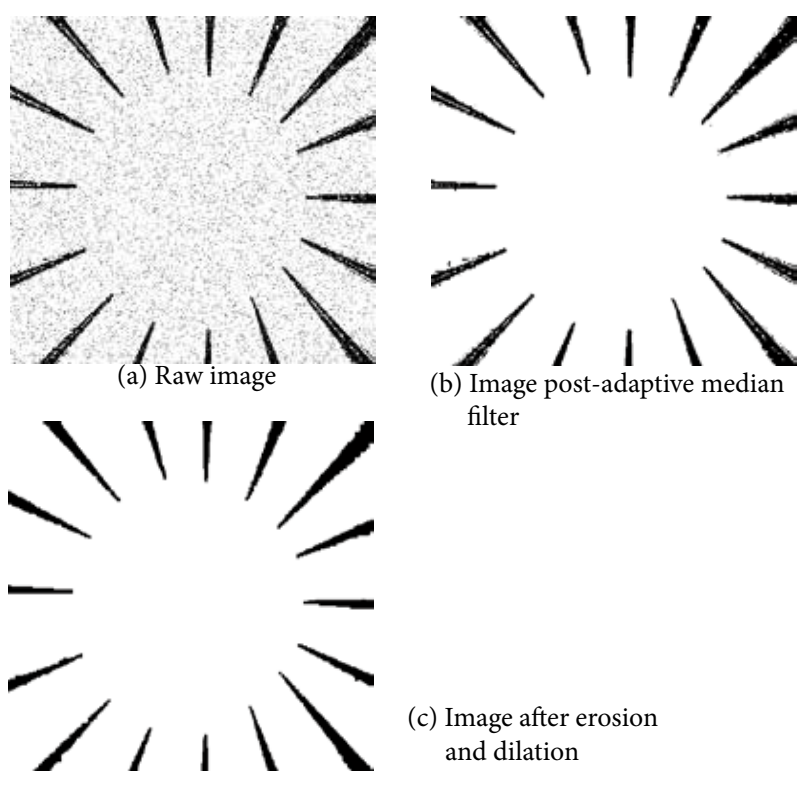

Figure 5. Pre-processing of image data. maximum gray scales are respectively $f_{\text {min }}, f_{\text {med }}$ and $f_{\text {max }}$. When $f_{\text {min }}<f_{\text {med }}<f_{\text {max }}, f_{\text {med }}$ is not a noise, and whether $f_{x y}$ is a noise can also be determined by $f_{\text {min }}<f_{x y}<f_{\text {max }}$. When neither fmed nor $f_{x y}$ is a pulse noise, $f_{x y}$ is preferred as the result (see Fig. 5(b)).

Since some hollow spots and rough edges are present in the image post-AMF, the erosion and dilation processes [13] are used to eliminate them. The rough edges are cleaned up by erosion, and the hollow spots are filled with dilation. The final result is shown in Fig. 5(c).

\subsection{Acquiring probe edges}

Even with the pre-processing, the silhouettes of the probes are still not smooth or straight as they should be. In this section, the probe edges are acquired by first drawing circles to find points on the edges, then fitting the points to straight lines.

Based on the image center, multiple circles can be drawn, each intersecting with all the probes. We define such a search circle as $c_{j}$, with $\mathrm{j}$ denoting the $j$-th circle starting from the inside. The probe in the top-center is denoted probe 1 , and the rest are numbered clockwise. Along a circle, the entry and exit points of a probe can be identified by the color values of the pixels. An entry point is denoted $d n_{1 j}$ (i.e., the entry point for the overlapping section between the $n$-th probe and the $j$-th circle), and an exit point is $d n_{2 j}$. All the probes can then be identified as seen in Fig. 6(a). Since all the probes are standard cones with straight edges, a line can be fitted from each edge, denoted $\ln _{i}$, with $i$ representing either the entry into or the exit from the circles.

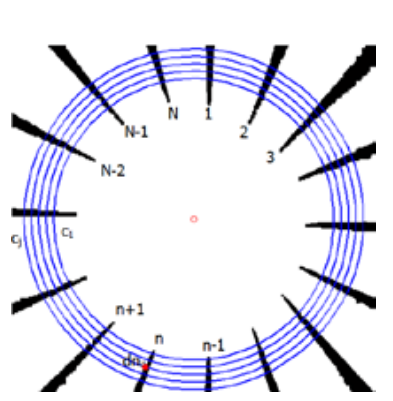

(a) Intersections of probes and search circles

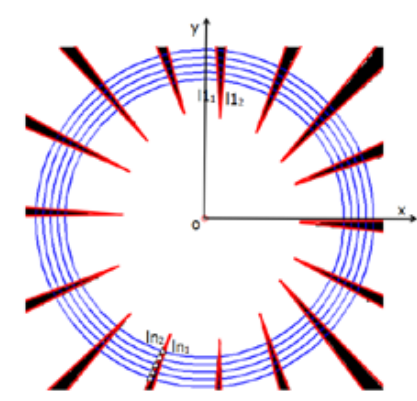

(b) Establishing Cartesian coordinates
Figure 6. Search circles.

Let the image center be the origin $O$, the width be the $x$-axis, and the height be the $y$-axis, a Cartesian coordinates system can be created as Fig. 6(b). The point $d n_{i j}$ has coordinates $\left(x n_{i j}, y n_{i j}\right)$, which have the following relationships: 


$$
\begin{gathered}
\left\{\begin{array}{l}
\bar{x} n_{i}=\sum_{j=1}^{J} x n_{i j} / J \\
\bar{y} n_{i}=\sum_{j=1}^{J} y n_{i j} / J
\end{array}\right. \\
\left\{\begin{array}{l}
k_{i}=\frac{\sum_{j=1}^{J}\left(\left|x n_{i j}-\bar{x} n_{i}\right|\right)\left(\left|y n_{i j}-\bar{y} n_{i}\right|\right)}{\sum_{j=1}^{J}\left(\left|x n_{i j}-\bar{x} n_{i}\right|\right)^{2}} \\
b n_{i}=\bar{y} n_{i}-k \bar{x} n_{i} \\
y n_{i}=k n_{i} x n_{i}+b n_{i}
\end{array}\right.
\end{gathered}
$$

where $J$ is the total number of search circles. Equation (3) is the equation of the line $\ln _{i}$.

\subsection{Determining the positions of the apices}

With the edges obtained, the apex of each probe can be pinpointed through their intersection, as seen in Fig. $7(a)$; the coordinates of these intersections are taken to be their accurate positions (see Fig. 7(b)). The apex of the $n$-th probe is denoted $D n(x n, y n)$.

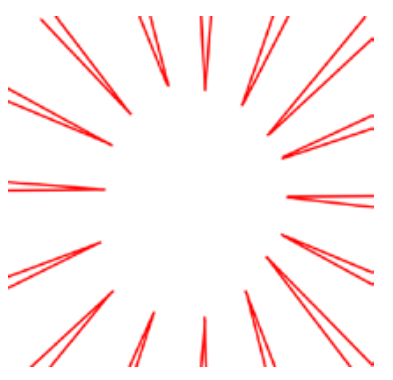

(a) Intersections of probe edges

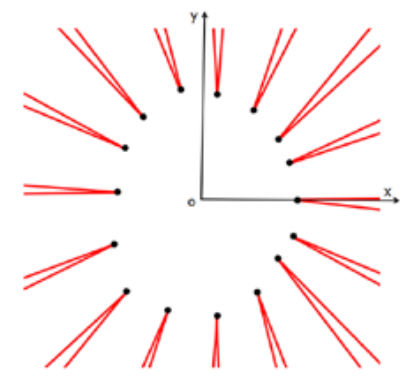

(b) Locations of probe apices
Figure 7. Locations of probe apices.

The coordinates $x n$ and $y n$ can be solved from the equations of the edges, as the following:

$$
\left\{\begin{array}{l}
x n=\frac{b n_{2}-b n_{1}}{k n_{1}-k n_{2}} \\
y n=\frac{k n_{2} b n_{1}-k n_{1} b n_{2}}{k n_{2}-k n_{1}}
\end{array}\right.
$$

\section{DETERMINING THE STRESS}

Using the integrated system, the stress along a borehole's cross-section can be determined. By measuring three intersecting boreholes, the three-dimensional stress at their intersection can then be calculated.

\subsection{Conversion between the borehole and the ground coordinates}

Determining three-dimensional stress requires knowing the angles between the boreholes and the ground. We begin by establishing a ground coordinate system $o x y z$, with the $x$-axis pointing east, the $y$-axis pointing north, the $z$-axis pointing up, and the origin $o$ as the center of the borehole cross-section at the measurement position. A borehole coordinate system $o x^{\prime} y^{\prime} z^{\prime}$ is also established, with $x^{\prime}$ in the $x y$ plane, $z^{\prime}$ pointing in the same direction as the axis of the borehole, and $y^{\prime}$ determined by the right-hand rule $[14,15]$, shown in Fig. 8.

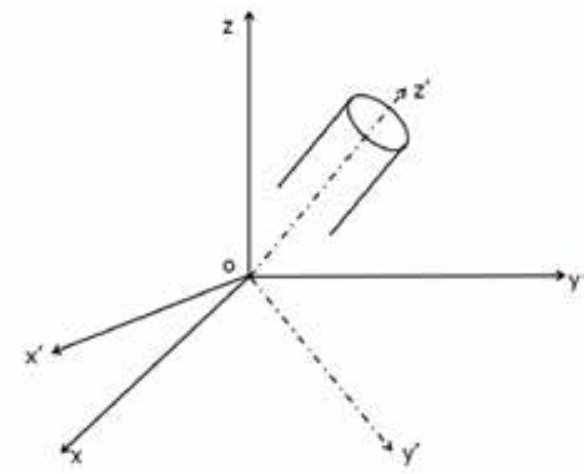

Figure 8. Ground and borehole coordinate systems.

Suppose both $o x y z$ and $o x^{\prime} y^{\prime} z^{\prime}$ are two Cartesian coordinate systems in an infinite space, the six 3-dimensional stress components $\sigma_{x}^{\prime}, \sigma_{y}^{\prime}, \sigma_{z}^{\prime}, \tau_{x y}^{\prime}, \tau_{y z}{ }^{\prime}, \tau_{z x}^{\prime}$ in $o x^{\prime} y^{\prime} z^{\prime}$ can be converted into their counterparts $\sigma_{x}, \sigma_{y}, \sigma_{z}, \tau_{x}, \tau_{y}, \tau_{z}$ in $o x y z$ with the following equations:

$$
\left\{\begin{aligned}
\sigma_{x}^{\prime}= & \sigma_{x} l_{1}^{2}+\sigma_{y} m_{1}^{2}+\sigma_{z} n_{1}^{2}+2 \tau_{x y} l_{1} m_{1}+2 \tau_{y z} m_{1} n_{1}+2 \tau_{z x} n_{1} l_{1} \\
\sigma_{y}^{\prime}= & \sigma_{x} l_{2}^{2}+\sigma_{y} m_{2}^{2}+\sigma_{z} n_{2}^{2}+2 \tau_{x y} l_{2} m_{2}+2 \tau_{y z} m_{2} n_{2}+2 \tau_{z x} n_{2} l_{2} \\
\sigma_{z}^{\prime}= & \sigma_{x} l_{3}^{2}+\sigma_{y} m_{3}^{2}+\sigma_{z} n_{3}^{2}+2 \tau_{x y} l_{3} m_{3}+2 \tau_{y z} m_{3} n_{3}+2 \tau_{z x} n_{3} l_{3} \\
\tau_{x y}^{\prime}= & \sigma_{x} l_{1} l_{2}+\sigma_{y} m_{1} m_{2}+\sigma_{z} n_{1} n_{2}+\tau_{x y}\left(l_{1} m_{2}+l_{2} m_{1}\right)+ \\
& +\tau_{y z}\left(m_{1} n_{2}+m_{2} n_{1}\right)+\tau_{z x}\left(n_{1} l_{2}+n_{2} l_{1}\right) \\
\tau_{y z}^{\prime}= & \sigma_{x} l_{2} l_{3}+\sigma_{y} m_{2} m_{3}+\sigma_{z} n_{2} n_{3}+\tau_{x y}\left(l_{2} m_{3}+l_{3} m_{2}\right)+ \\
& +\tau_{y z}\left(m_{2} n_{3}+m_{3} n_{2}\right)+\tau_{z x}\left(n_{2} l_{3}+n_{3} l_{2}\right) \\
\tau_{z x}^{\prime}= & \sigma_{x} l_{3} l_{1}+\sigma_{y} m_{3} m_{1}+\sigma_{z} n_{3} n_{1}+\tau_{x y}\left(l_{3} m_{1}+l_{1} m_{3}\right)+ \\
& +\tau_{y z}\left(m_{3} n_{1}+m_{1} n_{3}\right)+\tau_{z x}\left(n_{3} l_{1}+n_{1} l_{3}\right)
\end{aligned}\right.
$$

where $l_{1}, l_{2}$ and $l_{3}$ are, respectively, the cosines from $x^{\prime}, y^{\prime}$ and $z^{\prime}$ to $x ; m_{1}, m_{2}$ and $m_{3}$ are similar cosines from $x^{\prime}, y^{\prime}$ and $z^{\prime}$ to $y$; and $n_{1}, n_{2}$ and $n_{3}$ are cosines from $x^{\prime}, y^{\prime}$ and $z^{\prime}$ to $z$. 


\subsection{Relationship between the deformations and the 3-dimensional stress components}

When determining stresses we can base the global coordinate system on the earth, and establish a local system for each borehole. As long as we know the angles between the local systems and the global system, all the results from the local systems can be converted to the global system using Equation (5). Now we know the diameter of the borehole cross-section is deformed under stress, as shown in Fig. 9.

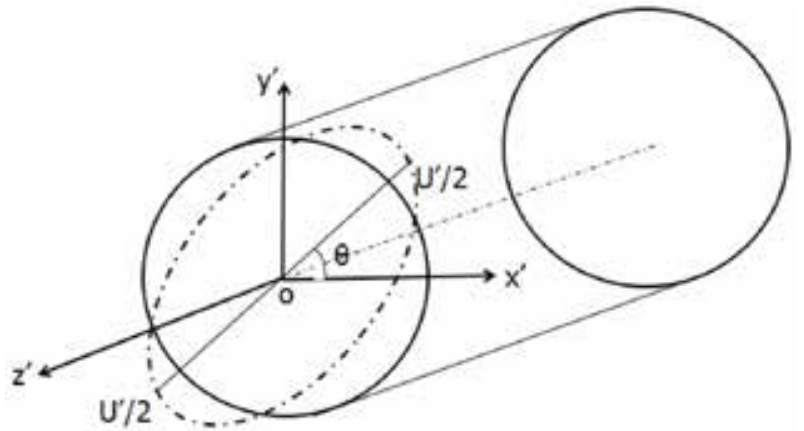

Figure 9. Deformation in the borehole coordinate system.

Assuming the borehole exists in a 3-dimensional, infinite frame, the following relationship can be found between the deformation $U^{\prime}$ and the 3-dimensional stress components:

$$
\begin{gathered}
U^{\prime}=\sigma_{x}^{\prime}\left(\frac{d}{E}\left[(1+2 \cos 2 \theta)\left(1-v^{2}\right)+d v^{2}\right]\right)+ \\
\sigma_{y}^{\prime}\left(\frac{d}{E}\left[(1-2 \cos 2 \theta)\left(1-v^{2}\right)+d v^{2}\right]\right)+\sigma_{z}^{\prime}\left(-\frac{d v}{E}\right)+\tau_{x y}^{\prime}\left(\frac{d}{E} 4(1-v) \sin 2 \theta\right)
\end{gathered}
$$

where $d$ is the borehole diameter; $\theta$ is the angle between the direction of the deformed diameter and $x^{\prime}$; rotating counterclockwise (CCW) from $x^{\prime}$ to the direction of the said diameter is positive; $E$ and $v$ are, respectively, the elastic modulus and Poisson's ratio of the rock at the measurement position; and $\sigma_{x}^{\prime}, \sigma_{y}^{\prime}, \sigma_{z}^{\prime}, \tau_{x y}^{\prime}$ can be converted to the global coordinate system via Equation (5).

\subsection{Equation of deformed cross-section}

After the stress is completed relieved, the borehole cross-section becomes elliptic in shape [16]. Under ideal conditions, the probe apices should also fall onto points on an ellipse, but since the borehole walls are not perfectly smooth, deviations and errors can still be present. Here, we approximate the ellipse with the leastsquares method.

Now the apices have coordinates $(x n, y n)$ with $n=1,2, \ldots$, $N, N$ being the total number of probes. The target function can be constructed:
$F(A, B, C, D, E)=\sum_{n=1}^{N}\left(x n^{2}+A x n \cdot y n+B y n^{2}+C x n+D y n+E\right)^{2}(7)$

We need to find the parameters $(A, B, C, D, E)$ that allow the apices $(x n, y n)$ to fall onto an ellipse as much as possible. This problem can be represented as obtaining the minimum value of $F(A, B, C, D, E)$. Based on the leastsquares theory, this implies the following relationships:

$$
\frac{\partial F}{\partial A}=\frac{\partial F}{\partial B}=\frac{\partial F}{\partial C}=\frac{\partial F}{\partial D}=\frac{\partial F}{\partial E}=0
$$

The following can be derived from Equation (8):

$$
\left[\begin{array}{ccccc}
\sum_{n=1}^{N} x n^{2} \cdot y n^{2} & \sum_{n=1}^{N} x n \cdot y n^{3} & \sum_{n=1}^{N} x n^{2} \cdot y n & \sum_{n=1}^{N} x n \cdot y n^{2} & \sum_{n=1}^{N} x n \cdot y n \\
\sum_{n=1}^{N} x n \cdot y n^{3} & \sum_{n=1}^{N} y n^{4} & \sum_{n=1}^{N} x n \cdot y n^{2} & \sum_{n=1}^{N} y n^{3} & \sum_{n=1}^{N} y n^{2} \\
\sum_{n=1}^{N} x n^{2} \cdot y n & \sum_{n=1}^{N} x n \cdot y n^{2} & \sum_{n=1}^{N} x n^{2} & \sum_{n=1}^{N} x n \cdot y n & \sum_{n=1}^{N} x n \\
\sum_{n=1}^{N} x n \cdot y n^{2} & \sum_{n=1}^{N} y n^{3} & \sum_{n=1}^{N} x n \cdot y n & \sum_{n=1}^{N} y n & \sum_{n=1}^{N} y n \\
\sum_{n=1}^{N} x n \cdot y n & \sum_{n=1}^{N} y n^{2} & \sum_{n=1}^{N} x n & \sum_{n=1}^{N} y n & N
\end{array}\right] .
$$

$$
\left[\begin{array}{l}
A \\
B \\
C \\
D \\
E
\end{array}\right]=-\left[\begin{array}{l}
\sum_{i=1}^{N} x n^{3} \cdot y n \\
\sum_{i=1}^{N} x n^{2} \cdot y n^{2} \\
\sum_{i=1}^{N} x n^{3} \\
\sum_{i=1}^{N} x n^{2} \cdot y n \\
\sum_{i=1}^{N} x n^{2}
\end{array}\right]
$$

Solving the five-order linear system results in $A, B, C, D$ and $E$, which are the parameters of an elliptical equation. For the ellipse, the center $\left(x_{0}, y_{0}\right)$, major axis $a$, minor axis $b$, and angle $\theta$ between the major axis and $x$ can then be determined as the following:

$$
\left\{\begin{array}{l}
\left(x_{0}, y_{0}\right)=\left(\frac{2 B C-A D}{A^{2}-4 B}, \frac{2 D-A D}{A^{2}-4 B}\right) \\
a=\sqrt{\frac{2\left(A C D-B C^{2}-D^{2}+4 B E-A^{2} E\right)}{\left(A^{2}-4 B\right)\left(B-\sqrt{A^{2}+(1-B)^{2}+1}\right)}} \\
b=\sqrt{\frac{2\left(A C D-B C^{2}-D^{2}+4 B E-A^{2} E\right)}{\left(A^{2}-4 B\right)\left(B+\sqrt{A^{2}+(1-B)^{2}+1}\right)}} \\
\theta=\tan ^{-1} \sqrt{\frac{a^{2}-b^{2} B}{a^{2} B-b^{2}}}
\end{array}\right.
$$




\subsection{Obtaining the stress data}

The system can measure the stress in a cross-section perpendicular to the borehole axis. But only three equations can be obtained from one borehole, which is not enough to solve for the six components in a 3 -dimensional system. To obtain the 3-dimensional stress data, the measurements must be made in three intersecting boreholes. The following uses one borehole as an example to show the process.

As in Fig. 10(a), the outline of the cross-section has changed from the circle before the stress relief to an ellipse. The deformation can be represented and compared using the outline's elliptical equation. From the origin $o$, three rays are drawn: the first along the positive $x$-axis, the second rotated CCW 60 degrees, and the third rotated CCW 120 degrees. The three rays, respectively, intersect with the pre-relief circle at $N_{1}, N_{2}$ and $N_{3}$, and with the post-relief ellipse at $M_{1}, M_{2}$ and $M_{3}$, with the coordinates $N_{i}\left(x_{n i}, y_{n i}\right)$ and $M_{i}\left(x_{m i}, y_{m i}\right)$, $i=1,2,3$. Therefore, the distance between each pair of points $\left|M_{i} N_{i}\right|$ is:

$$
\left|M_{i} N_{i}\right|=\sqrt{\left(x_{m i}-x_{n i}\right)^{2}+\left(y_{m i}-y_{n i}\right)^{2}} \quad(i=1,2,3)
$$

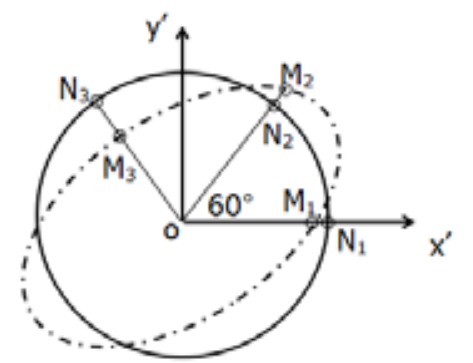

(a) Intersections of rays and the outline pre- and post-deformation

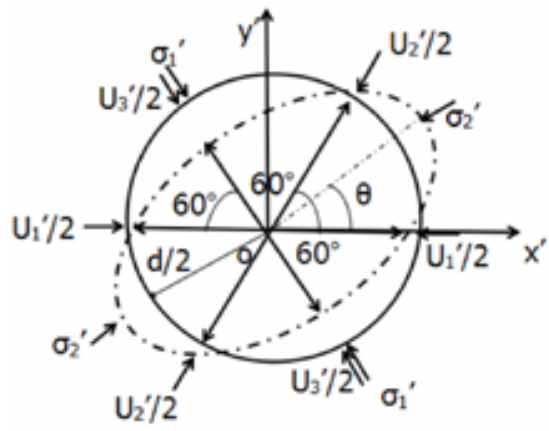

(b) Directions of deformation and stress

Figure 10. Deformation and state of stress in a cross-section perpendicular to the borehole.

Along the directions of the three rays, three diameter deformations $U_{1}^{\prime}, U_{2}^{\prime}$ and $U_{3}^{\prime}$ are defined as $2\left|M_{1} N_{1}\right|$, $2\left|M_{2} N_{2}\right|$ and $2\left|M_{3} N_{3}\right|$, rotated 60 degrees from each other, as shown in Fig. 10(b). Let $\beta$ be the angle between the first deformation $U_{1}^{\prime}$ and the maximum principal stress $\sigma_{1}^{\prime}$, and a CCW rotation from $U_{1}^{\prime}$ to $\sigma_{1}^{\prime}$ as positive, the state of stress, i.e., the amounts and directions of the stresses $\sigma_{1}^{\prime}$ and $\sigma_{2}^{\prime}$ can be computed from the three deformations:

$$
\left\{\begin{aligned}
& \sigma_{1}^{\prime}=\frac{E}{6 d\left(1-\mathrm{n}^{2}\right)} {\left[\left(U_{1}^{\prime}+U_{2}^{\prime}+U_{3}^{\prime}\right)+\frac{\sqrt{2}}{2}\right] \times } \\
& \times \sqrt{\left(U_{1}^{\prime}-U_{2}^{\prime}\right)^{2}+\left(U_{2}^{\prime}-U_{3}^{\prime}\right)^{2}+\left(U_{3}^{\prime}-U_{1}^{\prime}\right)^{2}} \\
& \sigma_{2}^{\prime}=\frac{E}{6 d\left(1-\mathrm{n}^{2}\right)}\left[\left(U_{1}^{\prime}+U_{2}^{\prime}+U_{3}^{\prime}\right)-\frac{\sqrt{2}}{2}\right] \times \\
& \quad \times \sqrt{\left(U_{1}^{\prime}-U_{2}^{\prime}\right)^{2}+\left(U_{2}^{\prime}-U_{3}^{\prime}\right)^{2}+\left(U_{3}^{\prime}-U_{1}^{\prime}\right)^{2}} \\
& \beta=\theta+\frac{\pi}{2}
\end{aligned}\right.
$$

where $v$ is the rock's Poisson's ratio and $E$ is its elastic modulus.

If we attach an axial strain gauge to the core obtained from the borehole, and apply a confining pressure to it, the ratio of its axial and radial strain is its Poisson's ratio. The elastic modulus can be obtained by using a borehole deformation gauge to measure its confining pressuredeformation curve, using the following equation:

$$
E=\frac{4 P_{0} r R^{2}}{U\left(R^{2}-r^{2}\right)}
$$

where $P_{0}$ is the confining pressure; $U$ is the average fine deformation caused by the confining pressure; $R$ and $r$ are, respectively, the outer and inner radii of the core.

If the axis of the borehole is along the same direction as a principal stress, the amount of which is already known, e.g., the gravity stress in a perfectly vertical well, measuring one borehole will be enough to determine the 3-dimensional state of stress at the measurement point.

\section{CASE STUDY}

We used the system to measure the stresses in the bottom outlets of a certain hydropower station. For the borehole ZK1 with diameter $168 \mathrm{~mm}$, the system performed the measurement operation along a depth of $150 \mathrm{~m}$, using a $91-\mathrm{mm}$ outer drill, and a $40-\mathrm{mm}$ inner drill. The cross-sectional stress data were obtained at 15 points, each separated by a depth interval of $10 \mathrm{~m}$. As an example, data from the first three points are analyzed below. 


\subsection{Solving for stress}

First, the system conducted data acquisition from depths 10,20 and $30 \mathrm{~m}$, generating images of probes before and after the stress relief. The images were processed by computer and stored as image data with directional information. The data then went under pre-processing, edge detection, and determination of probe apices.

With the positions of the probe apices known, they were fitted using least squares into circles and ellipses representing the statuses of the borehole before and after the stress relief. The circles or ellipses were described by equations, as shown in Fig. 11, Cartesian systems were established, and rays were drawn to calculate the three deformations $U_{1}^{\prime}, U_{2}^{\prime}$ and $U_{3}^{\prime}$ in each instance.

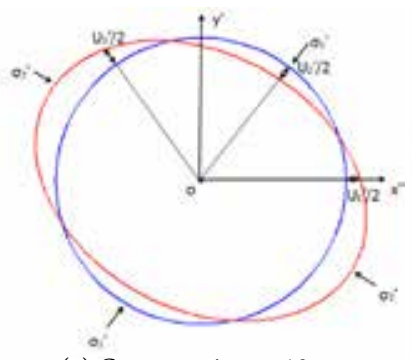

(a) Cross-section at $10 \mathrm{~m}$

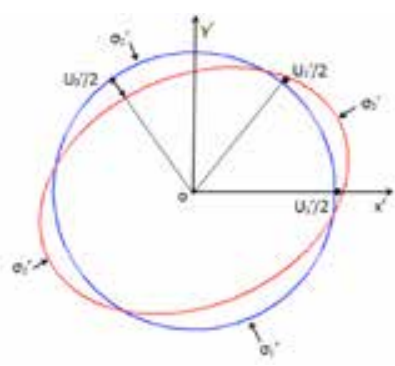

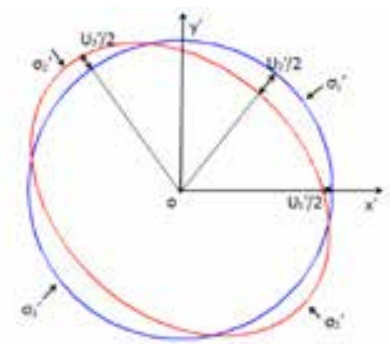

(b) Cross-section at $20 \mathrm{~m}$
Figure 11.

Cross-sections at depths 10,20 and $30 \mathrm{~m}$, pre- and post-stress relief.

Since the calculation requires knowing the rocks' Poisson ratios and elastic moduli, a RMT-150C rock testing machine was used to measure these parameters, with results shown in Table 1.

Table 1. Poisson's ratios and elastic moduli for cores at depths 10,20 and $30 \mathrm{~m}$.

\begin{tabular}{cccc}
\hline Core location & $10 \mathrm{~m}$ & $20 \mathrm{~m}$ & $30 \mathrm{~m}$ \\
\hline Elastic modulus $E / \mathrm{GPa}$ & 25.1 & 37.5 & 34.0 \\
\hline Poisson's ratio $v$ & 0.27 & 0.27 & 0.27 \\
\hline
\end{tabular}

The deformations $U_{1}^{\prime}, U_{2}^{\prime}$ and $U_{3}^{\prime}$ can then be used to determine the stresses in each cross-section using Equation (12), while the elliptical equation in each instance can be used to determine the angle $\theta$ between the major axis and the $x$-axis, thus obtaining the directions of two principal stresses for each measurement point. The results are shown in Table 2.

\subsection{Analyses of the result}

We conducted tests to validate the feasibility and reliability of the integrated system and the method. First, a three-axis compressor was used to perform a series of compression tests. For each core, confining pressures were applied that have the same amount and direction as the cross-sectional principal stresses above. Standard instruments were then used to measure the diameters of each hole before and after applying the pressures. The instrument is an inside micrometer with a precision of $0.001 \mathrm{~mm}$. The deformations caused by applying the known stresses were compared with the integrated system's results (see Fig. 12).

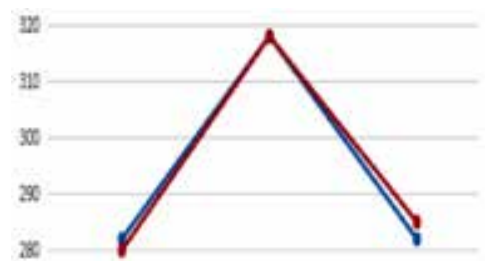

(a) Deformations of the $10 \mathrm{~m}$ core

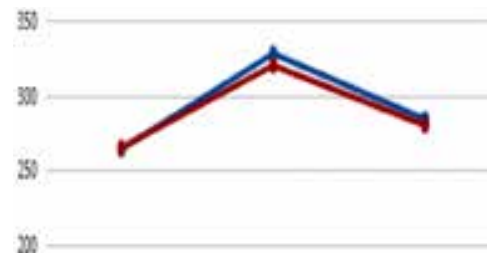

(b) Deformations of the $20 \mathrm{~m}$ core

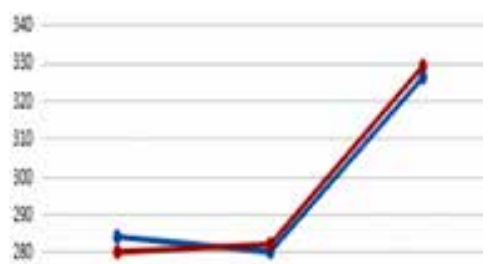

(c) Deformations of the $30 \mathrm{~m}$ core

(Blue: borehole measurements; Red: compression test results)

Figure 12. Comparison of deformations in borehole measurements and compression tests.

Table 2. Deformations and principal stresses along the cross-sections.

\begin{tabular}{c|ccc|cccc}
\hline \multirow{2}{*}{ Location } & \multicolumn{3}{|c|}{ Deformation } & \multicolumn{4}{c}{ Principal stresses along the cross-section } \\
\cline { 2 - 8 } & $U_{1}^{\prime} / \mu m$ & $U_{2}^{\prime} / \mu m$ & $U_{3}^{\prime} / \mu m$ & $\sigma_{1}^{\prime}$ amount & $\sigma_{1}^{\prime}$ direction & $\sigma_{2}^{\prime}$ amount & $\sigma_{2}^{\prime}$ direction \\
\hline $10 \mathrm{~m}$ & 282 & 318 & 282 & $9.1 \mathrm{MPa}$ & $\mathrm{N} 29.88^{\circ} \mathrm{E}$ & $1.0 \mathrm{MPa}$ & $\mathrm{S} 60.12^{\circ} \mathrm{E}$ \\
\hline $20 \mathrm{~m}$ & 264 & 328 & 284 & $14.3 \mathrm{MPa}$ & $\mathrm{N} 55.34^{\circ} \mathrm{E}$ & $1.5 \mathrm{MPa}$ & $\mathrm{S} 44.66^{\circ} \mathrm{E}$ \\
\hline $30 \mathrm{~m}$ & 287 & 280 & 326 & $10.7 \mathrm{MPa}$ & $\mathrm{N} 39.67^{\circ} \mathrm{W}$ & $1.3 \mathrm{MPa}$ & $\mathrm{S} 50.33^{\circ} \mathrm{W}$ \\
\hline
\end{tabular}




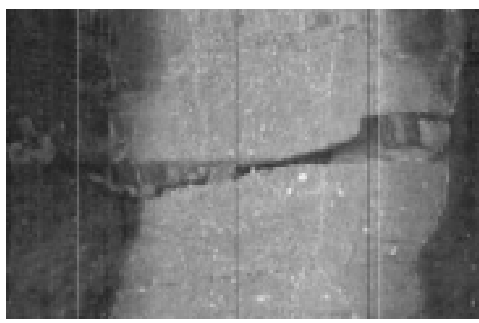

(a) $20 \mathrm{~m}$ depth

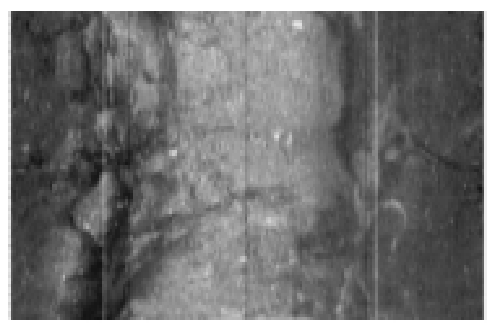

(b) $49 \mathrm{~m}$ depth

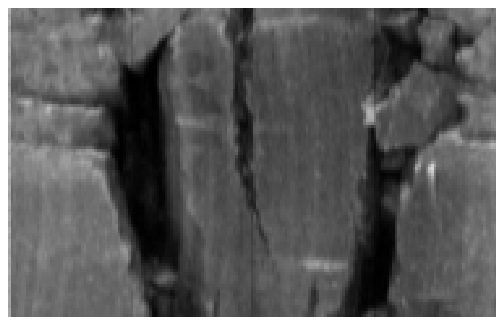

(c) $58 \mathrm{~m}$ depth

Figure 13. Partial borehole images.

The greatest variance between the two is $1.06 \%$ at $U_{3}^{\prime}$ in Fig. 12(a), 2.44\% at $U_{2}^{\prime}$ in Fig. 12 (b), and $2.44 \%$ at $U_{1}^{\prime}$ in Fig. 12(c). It can be seen that the general tendencies of the deformations are similar between the two groups of results, with errors below $2.5 \%$. Therefore, the system's measurements can be deemed valid.

The inner surfaces of a borehole might crack or peel off when the stress reaches a certain level. Such occurrences can also be used to determine the direction of the stress $[17,18,19]$. In our validation of the system, we used it to observe this borehole, and found the existence of cracks at $20 \mathrm{~m}$ (see Fig. 13(a)), and peeled off surfaces at $49 \mathrm{~m}$ and $58 \mathrm{~m}$ (see Figs. 13(b) and (c)). Their directions were consistent with calculations of the system, which were, respectively, along the west-east and north-south axes.
For the measurements of $\mathrm{ZK} 1$, the borehole was along the vertical direction. If we consider the geostress to be a principal stress, one measurement is enough to determine a point's 3-dimensional state of stress. Hence, the measurements over the full lengths of the borehole can be used to describe the vertical distribution of the stresses, which is depicted as rose diagrams in Table 3. The circumferential direction represents the directions of the stresses, and rotates clockwise from true north. The radial direction represents the amount of stress, with weaker stresses closer to the center, and stronger ones farther away from the center.

It can be seen in Table 3 that ZK1's maximum principal stress largely falls in the $10 \mathrm{MPa}$ range, existing along the west-east direction, while the minimum principal

Table 3. Summary of stress distribution in ZK1.

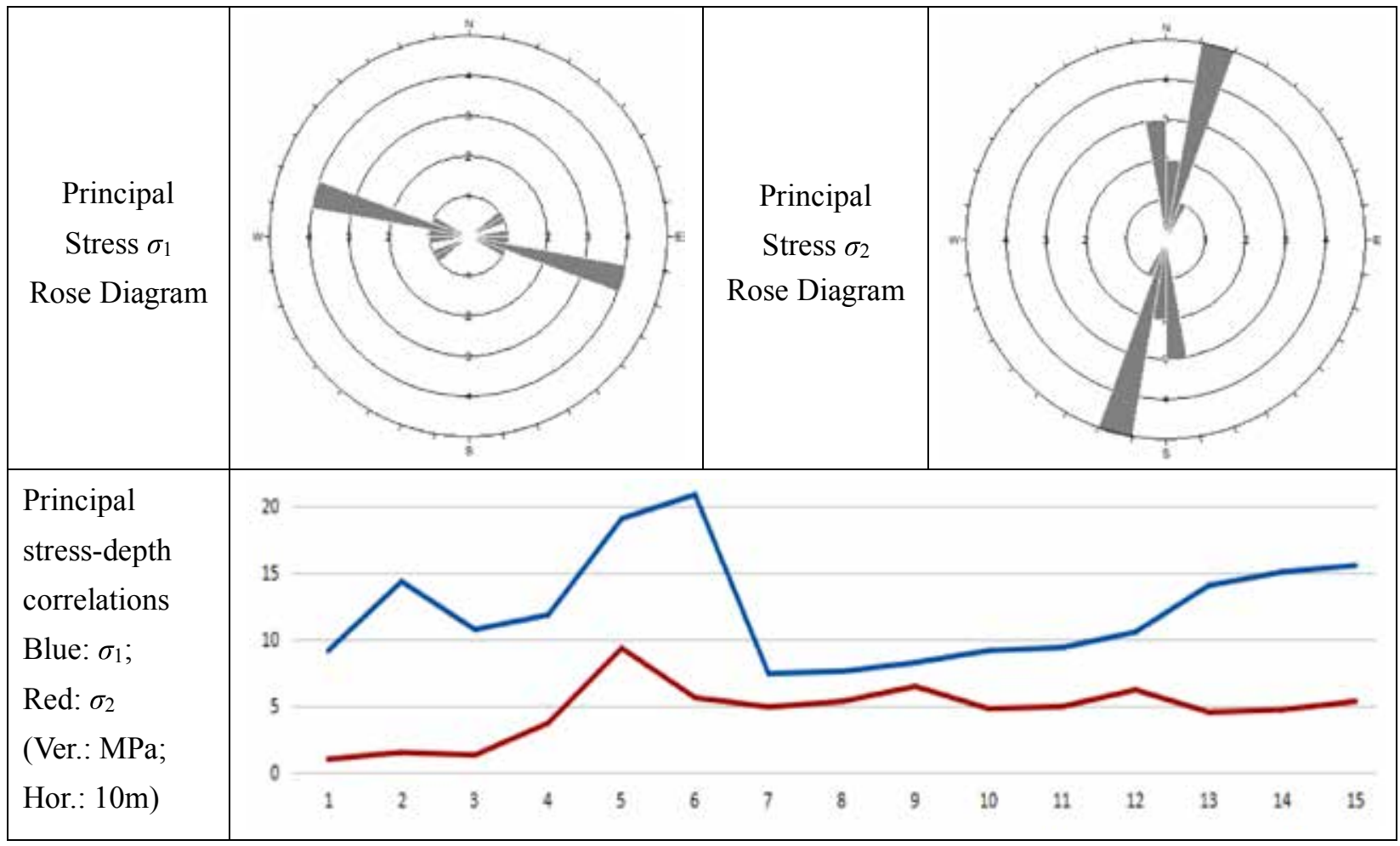


stress is around 1.3 MPa along the north-south direction. There is no linear correlation between the stress and depth; the stresses are affected by multiple factors, and vary greatly between the depths. The distribution of stress is at least partially stochastic. It can only be described using a unified equation when the distribution is normal or log-normal [20]. For this survey, linear interpolation is used to describe the stress within each $10 \mathrm{~m}$ segment between measurement points, and reduce the complexity of stochasticity. The stress at each depth value is assumed to be a linear function of the two measurement points before and after it.

The results show that the stress varies considerably with depth. We chose the maximum values as an intuitive way to describe the borehole's stress characteristics. The maximum values of the principal stresses $\sigma_{1}$ and $\sigma_{2}$ are used as the reference values of the stress in this borehole, while the reference directions are obtained by observing the rose diagram.

The measurements of ZK1 indicate the system can effectively acquire the borehole deformations along the well, avoiding changes caused by rock creep during the long periods of waiting, which leads to more accurate results. The system also enables more efficient measurements of greater depths. The method of stress determination based on the system is a good approach to describe the movements of the probes, which can be used to calculate the deformations and principal stresses. The resulting cross-sectional stresses from the depths can be used to provide an intuitive depiction of the borehole's stress variations using rose and contoured diagrams.

\section{CONCLUSION}

This paper proposes a measurement method for geostress based on an integrated drilling and optical microscopy system. It employs microscopic imaging and direct contact probes to capture the changes of a borehole's cross-sections before and after the stress relief. The resulting images are analyzed with search circles to obtain the positions of the probe apices, which can be fitted into ellipses that describe the cross-sectional outlines, and calculate the states of stress. The validity and accuracy of the method was verified by tests and field application in the ZK1 borehole. The results show that: (1) the integrated system avoids inaccuracies caused by time delays; (2) the integrated system can be used to measure micrometer-grade deformations; (3) the search circle approach can accurately obtain the positions of probe apices; (4) the stress-measurement method based on the system is accurate and feasible; (5) the method can be used for stress measurements in deep positions and complex environments.

\section{REFERENCES}

[1] Liu, Y. F. 2000. Rock Body Geostress and Construction Engineering [in Chinese], Hubei Science \& Technology, Wuhan.

[2] Ge, X.R., Hou, M.X. 2012. Principle of in-situ 3D rock stress measurement with borehole wall stress relief method and its preliminary applications to determination of in-situ rock stress orientation and magnitude in Jinping hydropower station. Sci China Tech Sci. 55, 939-949. DOI: 10.1007/s11431011-4680-x

[3] Worotnicki, G., Walton, R. 1976. Triaxial "hollow inclusion" gauges for determination of rock stresses in-situ. Investigation of Stress in Rock - Advances in Stress Measuremnent, Proc, Int, Symp, Sydney, 1-8 (supplement).

[4] Nur, A., Slmmons, G. 1969. Stress-induced velocity anisotropy in rock: an experimental study. J. of Geophys. Res. 74(27), 667 6674. DOI: 10.1029/ JB074i027p06667

[5] AI Kai, Han Xiao-yu, Li Yong-song 2006. Regression Analysis of In-situ Stress for Wudongde Hydropower Station, Jinsha River [in Chinese]. Chinese Journal of Underground Space and Engneering 2, (26), 930-933. DOI: 10.3969/j.issn.16730836.2006.06.011

[6] Xu, Z.L. 2006. Elastic Mechanics [in Chinese], China Higher Education, Beijing.

[7] Luo, C.H., Wang, W. 2003. Refined theory of generalized plane-stress problems in elasticity. Acta Mechanica Solida Sinica 24(02), 192-196. DOI: 10.3969/j.issn.0254-7805.2003.02.009

[8] Yoshikawa, S., Mogi, K. 1981. A new method for estimation of the crustal stress from cored rock samples: laboratory study in the case of uniaxial compression. Tectonophysics 74, 323-339. DOI: 10.1016/0040-1951(81)90196-7

[9] Cai, M.F., Qiao, L., Li, H.B. 1995. Theory and Techniques of Geostress Measurement [in Chinese]. China Science, Beijing.

[10] Amadei, B., Stephansson, O. 1997. Rock stress and its measurement, Chapman and Hall, London.

[11] Duncan Fama, M.E., Pender, M.J. 1980. Analysis of the hollow inclusion technique for measuring in-situ rock stress. Int. J. Rock Mech. Min. Sci. And Geomech. Abstr. 17, 137-146. DOI: 10.1016/01489062(80)91360-1

[12] Wang, L.J., Pan, L.Z. 1991. Geostress Measurement and Its Application in Engineering [in Chinese]. China Geology, Beijing.

[13] Zhang, Z., Xu, C. 2014. Digital Image Processing and Machine Vision, China Posts \& Telecom, Beijing. 
[14] Brudy, M., Zoback, M.D., Fuchs, K. et al. 1997. Estimation of the complete stress tensor to $8 \mathrm{~km}$ depth in the KTB scientific drill holes: implications for crustal strength. Journal of Geophysical Research 102(B8), 18453-18475. DOI: 10.1029/96JB02942

[15] Sun, W.C., Min, H., Wang, C.Y. 2008. Threedimensional geostress measurement and geomechanical analysis. Chinese Journal of Rock Mechanics and Engineering 27(z2), 3778-3784. DOI: $10.3321 /$ j.issn: 1000-6915.2008.z2.070

[16] Wang, C.Y., Han, Z.Q., Wang, J.C., Wang, Y.T. 2016. Study of borehole geometric shape features under plane stress state, Chinese Journal of Rock Mechanics and Engineering 35(z1), 2836-2846. DOI: $10.13722 /$ j.cnki.jrme.2015.1045

[17] Quan Jiang, Xia-ting Feng, Jing Chen, Ke Huang, Ya-li Jiang 2013. Estimating in-situ rock stress from spalling veins: A case study. Engineering Geology 152(1), 38-47. DOI: 10.1016/j. enggeo.2012.10.010

[18] Jiang, Q., Feng, X.T., Xiang, T.B., Su, G.S. 2010. Rockburst characteristics and numerical simulation based on a new energy index: a case study of a tunnel at $2500 \mathrm{~m}$ depth. Bulletin of Engineering Geology and the Environment 69, 381-388. DOI: 10.1007/s10064-010-0275-1

[19] Quan Jiang, Xia-ting Feng, Yilin Fan, Qixiang Fan, Guofeng Liu, Shufeng Pei 2017. In situ experimental investigation of basalt spalling. Tunnelling and Underground Space Technology 68, 82-94. DOI: 10.1016/j.tust.2017.05.020

[20] Jiang Q., Zhong S., Cui J., Feng X.T., Song, L. 2016. Statistical Characterization of the Mechanical Parameters of Intact Rock Under Triaxial Compression_An Experimental Proof of the Jinping Marble. Rock Mechanics \& Rock Engineering 49 (12), 4631-4646. DOI: 10.1007/s00603-0161054-5 